\title{
Crowd-powered Creativity Support Systems
}

\author{
Jonas Oppenlaender \\ jonas.oppenlaender@oulu.fi \\ University of Oulu \\ Oulu, Finland
}

\begin{abstract}
Crowdsourcing has great potential in supporting humans to be more creative. This doctoral dissertation explores crowdpowered creativity support systems and covers a research arc from the fundamental prerequisites of leveraging crowds for creativity support to an accompanying set of case studies to clarify how complex creative work can be supported in practice.
\end{abstract}

\section{CCS CONCEPTS}

- Human-centered computing $\rightarrow$ Interactive systems and tools; • Information systems $\rightarrow$ Crowdsourcing.

\section{KEYWORDS}

creativity support systems, crowdsourcing, creativity

\section{ACM Reference Format:}

Jonas Oppenlaender. 2020. Crowd-powered Creativity Support Systems. In ACM SIGCHI Symposium on Engineering Interactive Computing Systems (EICS '20 Companion), June 23-26, 2020, Sophia Antipolis, France. ACM, New York, NY, USA, 4 pages. https://doi.org/

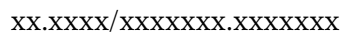

\section{INTRODUCTION}

Crowdsourcing has been applied in a great number of applications, from finding cures for lower back pain [7] to supporting weight loss [8]. Since humans excel in divergent thinking, recombination, analogical transfer, and other fundamental characteristics needed for creativity, crowdsourcing has great potential in supporting creativity [10]. Supporting human creativity has been considered as one of the grand challenges of human-computer interaction [26].

And indeed, many studies and experiments in academia and industry routinely tap into the creative ability of the crowd, either for creativity-directed research or for simply eliciting ideas and creative artifacts from a large group of people [6]. However, the ability and willingness of the crowd

Permission to make digital or hard copies of part or all of this work for personal or classroom use is granted without fee provided that copies are not made or distributed for profit or commercial advantage and that copies bear this notice and the full citation on the first page. Copyrights for third-party components of this work must be honored. For all other uses, contact the owner/author(s).

EICS '20 Companion, fune 23-26, 2020, Sophia Antipolis, France

(c) 2020 Copyright held by the owner/author(s).

ACM ISBN xxx- $\mathrm{x}-\mathrm{x} x \mathrm{xx}-\mathrm{xxxx}-\mathrm{x} / \mathrm{xx} / \mathrm{xx}$.

https://doi.org/xx.xxxx/xxxxxxx.xxxxxxx to participate in creative work and creative experiments is largely unquestioned by the academic community. Insights into the crowd workers' perspectives are rare, but important, as they may inform the design of studies with higher validity. It is imperative to develop an understanding of what crowd workers, as one of the primary stakeholder groups of crowdsourcing platforms, think about creative work. Such insights may inform the design of online systems and tools that rely on crowd-powered creativity [22,24]. Much is to be learned about how the crowd perceives creative tasks and experiments, and what could be done to optimize such studies.

The research is motivated by the growing trend towards automation and a concern about the effect of artificial intelligence (AI) on the future work force. Creativity is what makes us human, and machines fail to epitomize creativity. Machines can interact with us in human-like ways, but they cannot tell whether a photograph is aesthetically pleasing, whether a painting will elicit feelings, or whether there is a deeper meaning behind a creative artifact. Creativity is thus one of the key skills that will secure human's place in future value chains. Research on human-centered AI [27], interactive machine learning [5] with a "human in the loop", humanmachine-interaction [14], and hybrid AI [2] highlights the need of involvement of humans in future work flows, as a source of sentient human control in AI systems.

This research project explores the design space of crowdpowered creativity support systems [22] in two separate parts (see Figure 1). The first part (papers I-IV) investigates the crowd worker's perspective of creative work on crowdsourcing platforms. The findings from this part affect the requester's pre-ideation activities of outsourcing creative work (i.e., planning a crowdsourcing campaign, selecting the right crowdsourcing platform, and designing the task). The second part (papers V-VII) explores crowd-powered creativity support systems in three case studies.

\section{RELATED WORK}

Creativity. In this work, creativity is viewed as a domaingeneral concept. We follow the sociocultural view of creativity [1]. From this perspective, an idea or an artifact is considered creative if it is novel (or synonyms thereof, such as unique, original) and appropriate (or useful, effective) for a given domain, as judged by the domain's gatekeepers (the "field"). 


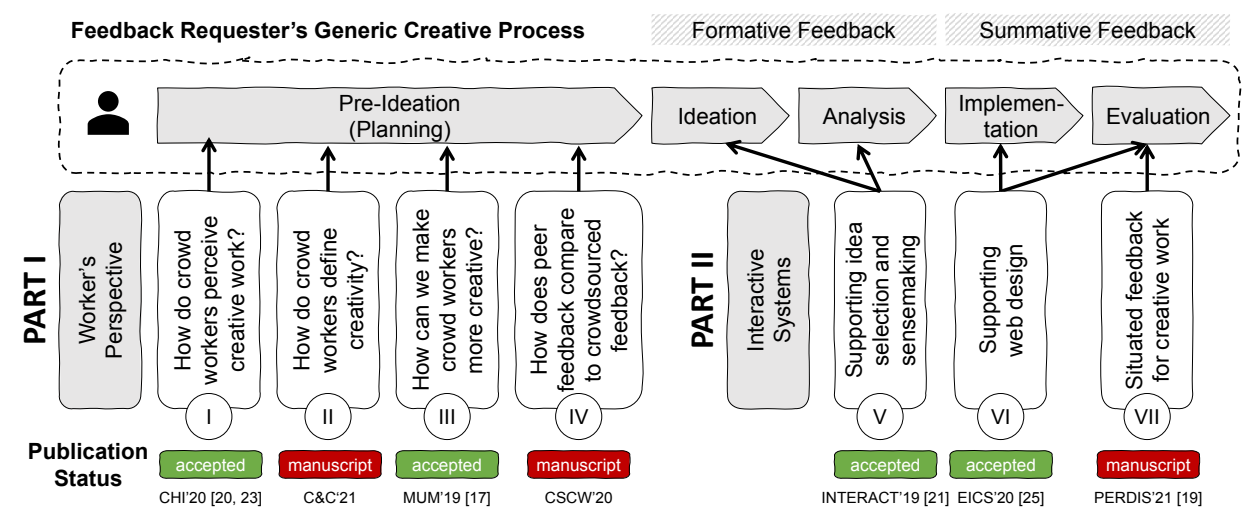

Figure 1: Structural overview of the research project.

Crowdsourcing. Crowdsourcing has become part of the toolkit of many researchers as a means for cost-effectively recruiting study participants and conducting experiments online. Our work focuses on investigating creativity on generalpurpose crowdsourcing platforms. On these platforms, requesters publish tasks for an anonymous crowd to complete in exchange for a small monetary reward [9]. Other crowdsourcing platforms created for the specific purpose of eliciting creative works and ideas, such as Teefury, 99Designs, and many others, are out of scope of this work.

Crowd Feedback for Creative Work. Crowd feedback systems are computer-mediated systems that enable creative individuals to collect feedback and critique from a large number of people online $[12,15,18,30]$. The present research project investigates the application of crowd feedback systems for supporting the creative process of the feedback requester. To this end, we adapt a generic model of the requester's creative process [31]: ideation (i.e., idea generation), analysis (e.g., idea selection and making sense of alternatives), implementation, and evaluation. In addition, we place emphasis on the early stages of the creative process, and extend the creative process model with pre-ideation activities, as found in the process typology in [6]. We acknowledge that this creative process is often iterative in practice and may evolve over time [3]. In this work, the generic process model serves as an overall framework along which we observe and investigate the crowd worker's perspectives in several creative activities.

\section{CROWDSOURCING CREATIVITY SUPPORT}

The remainder of this paper briefly presents completed studies (papers I and III) and systems (papers V and VI), followed by an outlook on future studies (papers II, IV and VII).

\section{How do Crowd Workers perceive Creative Work? (I)}

Resentment towards creative work and pre-exposure to common creative tasks could negatively impact the validity of creativity studies and crowd-powered creativity support systems. In this work [20, 23], we explored what crowd workers think about creativity on two general-purpose crowdsourcing platforms: Amazon Mechanical Turk and Prolific. To the best of our knowledge, our work contributed the first worker-centered qualitative study of creative work on generalpurpose crowdsourcing platforms.

The Study. We launched a survey task on the two crowdsourcing platforms. A mixed-method analysis of the workers' responses $(N=215)$ allowed us to develop complementary insights into creative work on the two platforms and a sense for different archetypal worker profiles.

Findings. Among other findings, we identified several archetypes of workers with different preferences and motivations for creative work. For instance, we found that professional crowd workers may have a negative attitude towards creative tasks. Different types of workers thus require different strategies for crowdsourcing creative work. Further, we found evidence that the overwhelming majority of workers $(90 \%)$ in our sample preferred to work alone. Collaborative work flows have been researched in prior literature and some were found to be superior to non-collaborative work. Our finding stresses the need for careful design of the task and experiment, as enforced cooperation may cause issues in crowd-powered creativity support systems. Last, we found that some generalpurpose crowdsourcing platforms may be more suitable for creative work than other platforms. The above findings highlight the need for novel mechanisms for recruiting workers interested in creative work.

\section{Augmenting Creativity of Crowd Workers (III)}

Microtask crowd work can be monotonous and repetitive and workers may not be "in the mood" for creative work. Prior research found that looking at a problem through the lens of a different person may lead individuals to be more creative [11, 13, 28]. In this work [17], we investigated the use of 
roles in stimulating the divergent thinking of individuals in two complementary studies.

The Studies. In the first study, we implemented an online instrument for priming workers with roles and images, and recruited crowd workers $(N=60)$ to complete a divergent thinking task while assuming a role. In the second study, we observed the effect of roles on the ideation process of individuals when they reach an impasse in the flow of ideas.

Findings. In contrast to prior work, our analysis could not confirm computational priming having a significant effect on the outcome of a small batch of creative tasks. Our findings highlight that adopting roles can be an effective tool when creative individuals run out of ideas, but roles are not a silver bullet for improving divergent thinking.

\section{CREATIVITY SUPPORT SYSTEMS}

This section provides a brief overview of two crowd-powered creativity support systems created in this research project.

\section{CrowdUI: Supporting Web Design (VI)}

CrowdUI [25] is a crowd-powered creativity support system that enables the community of a website to visually provide feedback to the designer of the website, using the website itself as a canvas. Website visitors can directly modify (move, delete, resize) the elements of the web page. CrowdUI's multistep process includes a tutorial to familiarize the user with the tool, as well as a peer review stage in which users rate other users' creations. The system allows designers to inspect the individual modifications of the user interface, and also provides further decision support by visually aggregating user modifications in heatmaps. The system was evaluated in a study with 45 users and 60 web developers with promising results.

\section{GAS: Supporting Search and Exploration (V)}

We designed and tested an intervention [21] to support writers in finding and exploring different ideas for a writing piece. The lightweight system draws on a crowdsourced database and supports complex searches for information, that is, searches with at least two criteria, through a faceted filtering interface. Our study compared the system to Google Search and found the system provides its users with support for exploring an information space and selecting ideas.

\section{FUTURE WORK}

The following three sections describe future work in the context of this research project.

\section{How do crowd workers define creativity? (II)}

We will provide an in-depth exploration into how crowd workers on general-purpose crowdsourcing platforms conceptualize creativity. A conceptual gap between how workers conceive creativity and how requesters of creative work (often implicitly) define creativity may potentially impact the validity of creativity studies and creativity support systems. Ideally, this conceptual gap should be minimized.

\section{Crowdsourced Feedback versus the Classroom (IV)}

We will investigate the perceived usefulness and fairness of crowdsourced feedback in the context of an undergraduate design course. The study will add to the body of knowledge of applying crowd feedback systems in education [4,29]. The aim of this study is to contextualize crowdsourced feedback and creativity support within a realistic design context.

\section{SIMPLEX: Situated Feedback on Public Displays (VII)}

While the willingness of situated workers to contribute to a task has been investigated (e.g., [16]), the willingness of a situated worker to provide summative feedback for creative artifacts has received little attention. With our third system, we will explore a situated system for supporting creative individuals with crowdsourced feedback for digital artifacts on public displays. The project aims to investigate the interplay and communication between feedback requesters and the situated crowd. In a preliminary needfinding study [19] with two artists and 12 users, we evaluated eight different types of feedback. We found a preference for simple feedback mechanisms on the side of the users, and identified the problem of making sense of many different feedback items as one of the key challenges for the requester of crowdsourced feedback.

\section{CONCLUSION}

Our work helps researchers and industry professionals who wish to harness the inherent convenience and power of crowdsourcing platforms in creativity-oriented studies and crowdpowered creativity support systems.

This research aims to advance our understanding of how complex and subjective creative work can be supported with a distributed non-expert crowd of anonymous workers. The work contributes towards defining crowd-powered creativity support systems as a research area within Human-Computer Interaction (HCI) and Engineering Interactive Computing Systems (EICS).

\section{REFERENCES}

[1] Mihaly Csikszentmihalyi. 1996. Creativity: Flow and the Psychology of Discovery and Invention. Harper Collins Publishers, New York, NY, USA.

[2] Gianluca Demartini, Djellel Eddine Difallah, Ujwal Gadiraju, and Michele Catasta. 2017. An Introduction to Hybrid Human-Machine Information Systems. Found. Trends Web Sci. 7, 1 (2017), 1-87. https://doi.org/10.1561/1800000025

[3] Kees Dorst and Nigel Cross. 2001. Creativity in the design process: co-evolution of problem-solution. Design Studies 22, 5 (2001), 425-437. https://doi.org/10.1016/S0142-694X(01)00009-6

[4] Steven Dow, Elizabeth Gerber, and Audris Wong. 2013. A Pilot Study of Using Crowds in the Classroom. In Proceedings of the SIGCHI Conference 
on Human Factors in Computing Systems (CHI '13). ACM, New York, NY, USA, 227-236. https://doi.org/10.1145/2470654.2470686

[5] Jerry Alan Fails and Dan R. Olsen. 2003. Interactive Machine Learning. In Proceedings of the 8th International Conference on Intelligent User Interfaces (Miami, Florida, USA) (IUI '03). ACM, New York, NY, USA, 39-45. https://doi.org/10.1145/604045.604056

[6] Jonas Frich, Lindsay MacDonald Vermeulen, Christian Remy, Michael Mose Biskjaer, and Peter Dalsgaard. 2019. Mapping the Landscape of Creativity Support Tools in HCI. In Proceedings of the 2019 CHI Conference on Human Factors in Computing Systems (CHI '19). ACM, New York, NY, USA, Article 389, 18 pages. https://doi.org/10.1145/3290605.3300619

[7] Simo Hosio, Jaro Karppinen, Niels van Berkel, Jonas Oppenlaender, and Jorge Goncalves. 2018. Mobile Decision Support and Data Provisioning for Low Back Pain. Computer 51, 8 (2018), 34-43. https://doi.org/10.1109/MC.2018.3191250

[8] Simo Hosio, Niels van Berkel, Jonas Oppenlaender, and Jorge Goncalves. 2020. Crowdsourcing Personalized Weight Loss Diets. Computer 53 , 1 (2020), 63-71. https://doi.org/10.1109/MC.2019.2902542

[9] Jeff Howe. 2006. The Rise of Crowdsourcing. Wired Magazine 14, 6 (2006), 1-4.

[10] Aniket Kittur. 2010. Crowdsourcing, Collaboration and Creativity. XRDS 17, 2 (2010), 22-26. https://doi.org/10.1145/1869086.1869096

[11] Sheena Lewis, Mira Dontcheva, and Elizabeth Gerber. 2011. Affective Computational Priming and Creativity. In Proceedings of the SIGCHI Conference on Human Factors in Computing Systems (CHI '11). ACM, New York, NY, USA, 735-744. https://doi.org/10.1145/1978942.1979048

[12] Kurt Luther, Jari-Lee Tolentino, Wei Wu, Amy Pavel, Brian P. Bailey, Maneesh Agrawala, Björn Hartmann, and Steven P. Dow. 2015 Structuring, Aggregating, and Evaluating Crowdsourced Design Critique. In Proceedings of the 18th ACM Conference on Computer Supported Cooperative Work \& Social Computing (CSCW '15). ACM, New York, NY, USA, 473-485. https://doi.org/10.1145/2675133.2675283

[13] Robert R. Morris, Mira Dontcheva, and Elizabeth M. Gerber. 2012. Priming for Better Performance in Microtask Crowdsourcing Environments. IEEE Internet Computing 16, 5 (2012), 13-19. https://doi.org/10.1109/MIC.2012.68

[14] Donald A. Norman. 1994. How Might People Interact with Agents. Commun. ACM 37, 7 (1994), 68-71. https://doi.org/10.1145/176789.176796

[15] Jonas Oppenlaender. 2019. Supporting Creative Workers with Crowdsourced Feedback. In Proceedings of the 2019 ACM Conference on Creativity \& Cognition (C\&C '19). ACM, New York, NY, USA, 646-652. https://doi.org/10.1145/3325480.3326556

[16] Jonas Oppenlaender, Kennedy Opoku Asare, and Simo Hosio. 2018. CampusTracker: Assessing Mobile Workers' Momentary Willingness to Work on Paid Crowdsourcing Tasks. In Proceedings of the 2018 ACM International foint Conference and 2018 International Symposium on Pervasive and Ubiquitous Computing and Wearable Computers (UbiComp '18). ACM, New York, NY, USA, 648-653. https://doi.org/10.1145/3267305.3267550

[17] Jonas Oppenlaender and Simo Hosio. 2019. Design Recommendations for Augmenting Creative Tasks with Computational Priming. In Proceedings of the 18th International Conference on Mobile and Ubiquitous Multimedia (MUM '19). ACM, New York, NY, USA, Article 35, 13 pages. https://doi.org/10.1145/3365610.3365621

[18] Jonas Oppenlaender and Simo Hosio. 2019. Supporting Creative Work with Crowd Feedback Systems. In Proceedings of the $D C^{2} S^{2}$ Workshop on Designing Crowd-powered Creativity Support Systems (CHI '19). http://arxiv.org/abs/2004.09204

[19] Jonas Oppenlaender and Simo Hosio. 2019. Towards Eliciting Feedback for Artworks on Public Displays. In Proceedings of the 2019 ACM
Conference on Creativity \& Cognition (C\&C'19). ACM, New York, NY, USA, 562-569. https://doi.org/10.1145/3325480.3326583

[20] Jonas Oppenlaender and Simo Hosio. 2020. What Do Crowd Workers Think About Creative Work?. In Proceedings of the Workshop on Worker-Oriented Design: Expanding HCI Methods for Supporting Labor (CHI '20). http://arxiv.org/abs/2002.10887

[21] Jonas Oppenlaender, Elina Kuosmanen, Jorge Goncalves, and Simo Hosio. 2019. Search Support for Exploratory Writing. In HumanComputer Interaction - INTERACT 2019 (LNCS 11748), David Lamas, Fernando Loizides, Lennart Nacke, Helen Petrie, Marco Winckler, and Panayiotis Zaphiris (Eds.). Springer, Cham, Switzerland, 314-336. https://doi.org/10.1007/978-3-030-29387-1_18

[22] Jonas Oppenlaender, Maximilian Mackeprang, Abderrahmane Khiat, Maja Vukovic, Jorge Goncalves, and Simo Hosio. 2019. DC ${ }^{2} S^{2}$ : Designing Crowd-powered Creativity Support Systems. In Extended Abstracts of the 2019 CHI Conference on Human Factors in Computing Systems (CHI EA '19). Article W06, 8 pages. https://doi.org/10.1145/3290607.3299027

[23] Jonas Oppenlaender, Kristy Milland, Aku Visuri, Panos Ipeirotis, and Simo Hosio. 2020. Creativity on Paid Crowdsourcing Platforms. In Proceedings of the 2020 CHI Conference on Human Factors in Computing Systems (CHI '20). ACM, New York, NY, USA, 1-14. https://doi.org/10.1145/3313831.3376677

[24] Jonas Oppenlaender, Naghmi Shireen, Maximilian Mackeprang, Halil Erhan, Jorge Goncalves, and Simo Hosio. 2019. Crowd-powered Interfaces for Creative Design Thinking. In Proceedings of the 2019 ACM Conference on Creativity and Cognition (C\&C '19). ACM, New York, NY, USA, 722-729. https://doi.org/10.1145/3325480.3326553

[25] Jonas Oppenlaender, Thanassis Tiropanis, and Simo Hosio. 2020. CrowdUI: Supporting Web Design with the Crowd. Proceedings of the ACM on Human-Computer Interaction 4, EICS, Article 76 (2020), 28 pages. https://doi.org/10.1145/3394978

[26] Ben Shneiderman. 2009. Creativity Support Tools: A Grand Challenge for HCI Researchers. In Engineering the User Interface: From Research to Practice, Miguel Redondo, Crescencio Bravo, and Manuel Ortega (Eds.). Springer, London, UK, 1-9. https://doi.org/10.1007/978-1-84800-136-7_1

[27] Ben Shneiderman. 2020. Human-Centered Artificial Intelligence: Reliable, Safe \& Trustworthy. International fournal of Human-Computer Interaction 36, 6 (2020), 495-504. https://doi.org/10.1080/10447318.2020.1741118

[28] Jaime Teevan and Lisa Yu. 2017. Bringing the Wisdom of the Crowd to an Individual by Having the Individual Assume Different Roles. In Proceedings of the 2017 ACM SIGCHI Conference on Creativity and Cognition (C\&C '17). ACM, New York, NY, USA, 131-135. https://doi.org/10.1145/3059454.3059467

[29] Helen Wauck, Yu-Chun (Grace) Yen, Wai-Tat Fu, Elizabeth Gerber, Steven P. Dow, and Brian P. Bailey. 2017. From in the Class or in the Wild? Peers Provide Better Design Feedback Than External Crowds. In Proceedings of the 2017 CHI Conference on Human Factors in Computing Systems (CHI '17). ACM, New York, NY, USA, 5580-5591. https://doi.org/10.1145/3025453.3025477

[30] Anbang Xu, Shih-Wen Huang, and Brian Bailey. 2014. Voyant: Generating Structured Feedback on Visual Designs Using a Crowd of Non-experts. In Proc. Conf. Computer Supported Cooperative Work \& Social Computing (Baltimore, MD) (CSCW'14). ACM, New York, NY, 1433-1444. https://doi.org/10.1145/2531602.2531604

[31] Liang Zeng, Robert W. Proctor, and Gavriel Salvendy. 2011. Can Traditional Divergent Thinking Tests be Trusted in Measuring and Predicting Real-world Creativity? Creativity Research fournal 23, 1 (2011), 24-37. https://doi.org/10.1080/10400419.2011.545713 